\title{
High Order Mimetic Finite Difference Operators Satisfying a Gauss Divergence Theorem
}

Corbino $\mathrm{J}$ and Castillo JE*

Computational Science Research Center, San Diego State University, San Diego, CA, USA

\begin{abstract}
High order mimetic finite difference operators that satisfy a discrete extended Gauss Divergence theorem are presented. These operators have the same order of accuracy in the interior as well as the boundary, no free parameters and optimal bandwidth. They are constructed on staggered grids, using weighted inner products with a diagonal norm. We present several examples to demonstrate that mimetic finite difference schemes using these operators produce excellent results.
\end{abstract}

Keywords: Mimetic; Finite-difference; Castillo-Grone method; Differential operators; High-order

AMS subject classifications: 65D25, 65M06, 65N06

\section{Introduction}

Mimetic finite differences method have been experiencing a great deal of development in the last 10 years [1-5] and many applications of these methods have been reported in the literature. High order mimetic finite differences can be traced back to the work of Kreiss and Scherer [1], where they presented the Summation by Parts method (SBP). From their work, it is known that the order of accuracy at the boundary cannot be increased, with standard inner products, on nodal grids. They constructed a high order SBP operator, increasing the order of accuracy at the boundary [6-15], with a weighted inner product, on nodal grids. This operator was two orders less accurate at the boundary than the interior of the domain, on a nodal grid, with a diagonal weight matrix norm. In 2003, Castillo and Grone [7] using weighted inner products and staggered grids, constructed high-order divergence and gradient mimetic finite differences operators with the same order of accuracy in the interior as well as the boundary. These operators (CG) have been extended to higher dimensions and have been used very successfully in several applications [16-25]. However, these discrete operators have a set of free parameters and not necessarily the optimum bandwidth. In this paper, we construct high-order mimetic finite differences operators' divergence and gradient, on staggered grids, with diagonal weight matrix norms, no free parameters and optimal bandwidth. Some examples comparing the CG operators with the ones presented, exhibit results clearly showing that the new operators produce better results than that of the CG ones and in the worst case they produce the same ones.

This paper is organized as follows: We give a brief description of mimetic operators along with their properties, and the staggered grids along with a 3-D cell for illustration. We show how to construct the one dimensional second order mimetic gradient operator and show the gradient and divergence for the fourth order case. Note that for the second order case these operators are the same as the CG ones, but this only happens for the second order case, for fourth order and higher the new operators are different from the CG ones. We describe how to compute the weights for the inner products for the fourth order gradient and divergence mimetic finite difference operators. We show how to construct the operators in higher dimensions using Kronecker products as well as a mimetic finite difference Laplacian in one, two and three dimensions as well and the mimetic finite difference curl operator.
We show how these operators can be implemented in a compact form, minimizing the size of the stencils. We present examples that clearly demonstrate the new operators produce better results that the CG ones.

\section{Mimetic Operators}

Mimetic finite difference operators, divergence (D), gradient (G), curl (C) and laplacian (L) are discrete analogs of their corresponding continuum operators. These mimetic finite difference operators satisfy in the discrete sense the vector identities that the continuum ones do making them more faithful to the physics.

\section{Basic properties}

Mimetic operators $\left(\mathbf{G} \equiv \nabla, \quad \mathbf{D} \equiv \nabla, \quad \mathbf{C} \equiv \nabla \times\right.$ and $\left.\quad \mathbf{L} \equiv \nabla^{2}\right)$ fulfill the following:

$$
\begin{aligned}
& \mathbf{G} f_{\text {const }} 0, \\
& \mathbf{D} v_{\text {const }} 0, \\
& \mathbf{C G} f=0, \\
& \mathbf{D C} v=0, \\
& \mathbf{D G} f=\mathbf{L} f .
\end{aligned}
$$

In addition, while providing a uniform order of accuracy, CG operators satisfy:

$$
\langle\mathbf{D} v, f\rangle_{Q_{+}}\langle\mathbf{G} f, v\rangle_{P}=\langle\mathbf{B} v, f\rangle
$$

which is a discrete analogue of the extended Gauss divergence theorem [8], here $\mathbf{B}$ is called the mimetic boundary operator. From eqn. (6) we obtain:

$$
\langle Q \mathbf{D} v\rangle f+\langle P \mathbf{G} f, v\rangle=\langle\mathbf{B} v, f\rangle,
$$

*Corresponding author: Castillo JE, Computational Science Research Center, San Diego State University, San Diego, CA, USA, Tel: 6198826087; E-mail johnnycorbino@gmail.com

Received December 28, 2017; Accepted January 15, 2018; Published January 22, 2018

Citation: Corbino J, Castillo JE (2018) High Order Mimetic Finite Difference Operators Satisfying a Gauss Divergence Theorem. J Appl Computat Math 7: 387. doi: 10.4172/2168-9679.1000387

Copyright: $\odot 2018$ Corbino J, et al. This is an open-access article distributed unde the terms of the Creative Commons Attribution License, which permits unrestricted use, distribution, and reproduction in any medium, provided the original author and source are credited. 


$$
\begin{aligned}
& \left.\langle Q \mathbf{D} v\rangle+\mathbf{G}^{T} P v, f\right\rangle=\langle\mathbf{B} v, f\rangle, \\
& Q \mathbf{D} v+\mathbf{G}^{T} P v=\mathbf{B} v, \\
& Q \mathbf{D}+\mathbf{G}^{T} P=\mathbf{B} .
\end{aligned}
$$

\section{Staggered grids}

CG mimetic operators are defined over staggered grids. In this type of grids, scalar variables are stored at the centers of the cells; while vector components are placed at the edges (or faces, in 3D). In the following figures, $m, n$ and $o$ represent the number of cells along the $\mathrm{x}$-, $\mathrm{y}$ - and $\mathrm{z}$-axes, respectively (Figures 1-4).

\section{D Operators}

This section is focused on the construction of one-dimensional mimetic gradient and divergence operators. One-dimensional operators can be visualized as follows:

In Figure 5, $A$ and $A^{\prime}$ are sub matrices that approximate the derivatives at the left, and at the right boundary, respectively. $\operatorname{Dim}(A)=\operatorname{Dim}\left(A^{\prime}\right)$, and $A^{\prime}$ is a permutation of $A$. $M$ is a banded matrix of width $k$ (order of accuracy) that approximates the derivatives at the

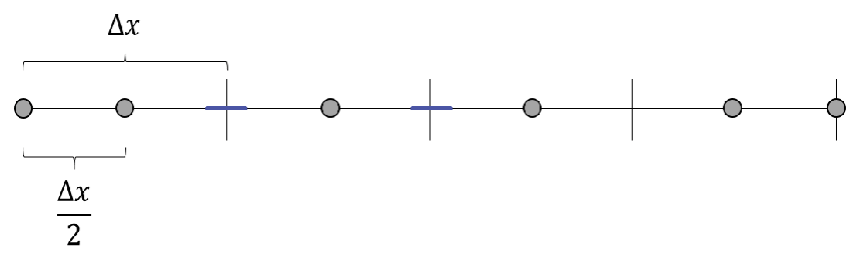

Figure 1: One-dimensional, uniform staggered grid. $\mathrm{m}=4$.

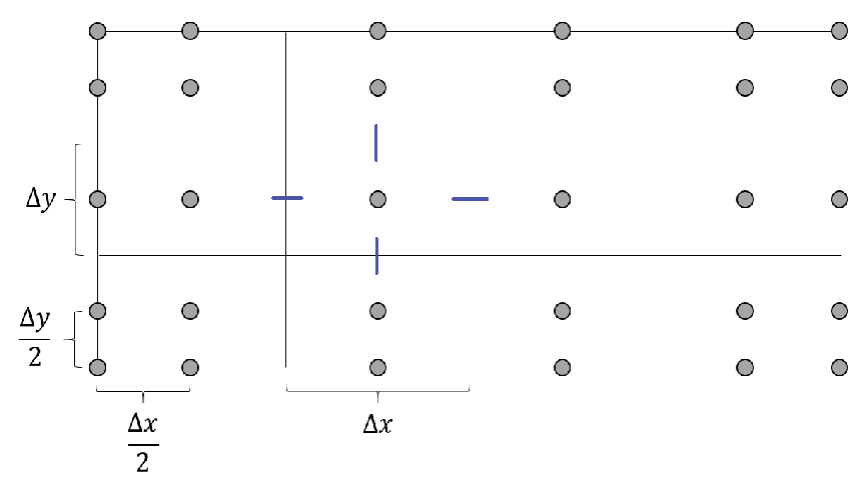

Figure 2: Two-dimensional, uniform staggered grid. $m=4$ and $n=3$.

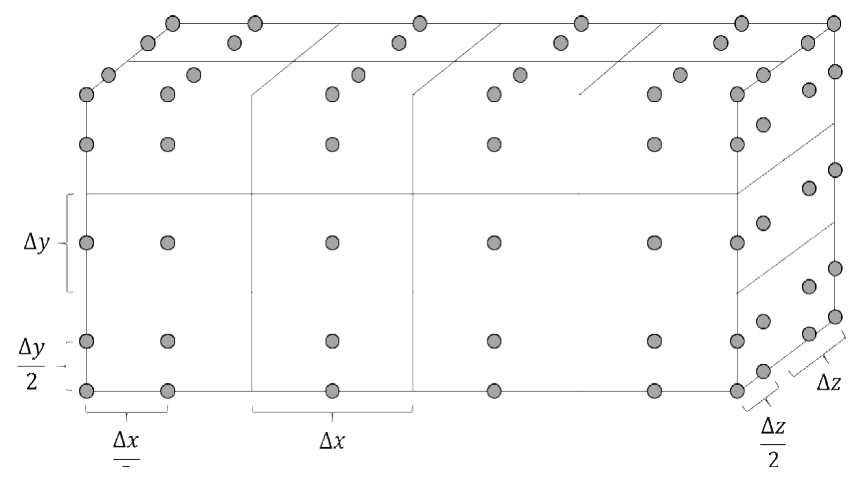

Figure 3: Three-dimensional, uniform staggered grid. $m=4, n=3$ and $o=2$.

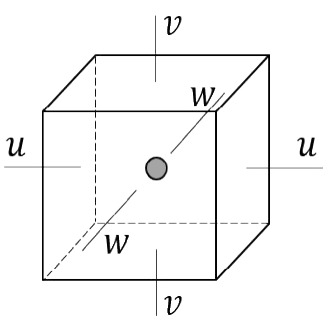

Figure 4: $A 3 D$ cell. $u, v$ and $w$ are the vector components used to compute the divergence. In case of the curl, we use the components that are tangential to the faces of the cell.

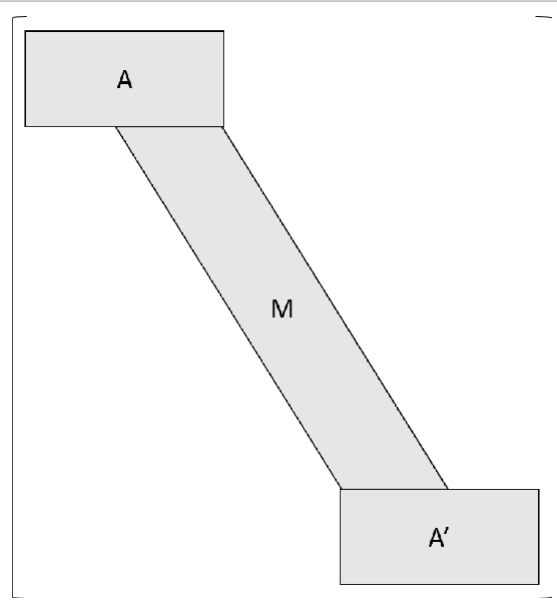

Figure 5: Taxonomy of 1D operator.

inner cells. The dimensions of $A$ depend on the type of operator and the desired order of accuracy.

\section{Gradient}

To construct a $k$ th-order mimetic gradient operator we need at least $2 k$ cells $(m \geq 2 k)$ so that there is no overlapping between $A$ and $A^{\prime}$.

$A$ and $A^{\prime}$ will have dimensions $\frac{k}{2}$ by $k+1$. We proceed to construct a Vandermonde matrix from the stencil (Figure 6), then, our "generator" vector is:

$$
\left[0 \frac{1}{2} \frac{3}{2}\right]
$$

and the corresponding Vandermonde matrix,

$$
\left[\begin{array}{lll}
0 & \frac{1}{4} & \frac{9}{4} \\
0 & \frac{1}{2} & \frac{3}{2} \\
1 & 1 & 1
\end{array}\right]
$$

Finally, we construct a right-hand side vector that only contains a ' 1 ' aligned to the second to last row of the matrix, producing the following system of linear equations:

$$
\left[\begin{array}{lll}
0 & \frac{1}{4} & \frac{9}{4} \\
0 & \frac{1}{2} & \frac{3}{2} \\
1 & 1 & 1
\end{array}\right]\left[\begin{array}{l}
x_{1} \\
x_{2} \\
x_{3}
\end{array}\right]=\left[\begin{array}{l}
0 \\
1 \\
0
\end{array}\right]
$$




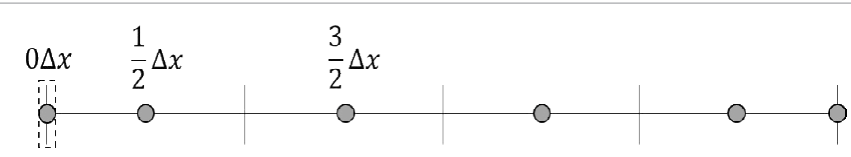

Figure 6: Stencil to compute a $2^{\text {nd }}$-order mimetic gradient at the left boundary. The segmented rectangle on the left represents the location of the value being calculated.

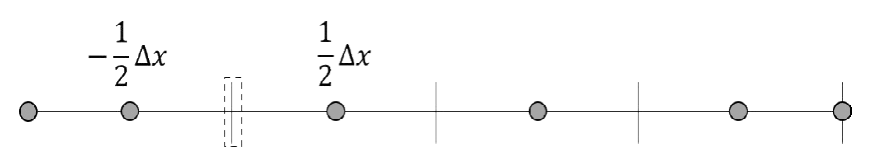

Figure 7: Inner stencil for $2^{\text {nd }}$-order mimetic gradient. Again, the segmented rectangle represents the location of the value being calculated

and from the solution $\left[x_{1}, x_{2}, x_{3}\right]=\left[\frac{-8}{3} 3 \frac{-1}{3}\right]$, we obtain the first row of our matrix $A$. To obtain the successive rows of $A$ we just need to "shift" the stencil to the right $\frac{k}{2}-1$ time and is computed as follows:

$$
A^{0=}-P_{p} A P_{q}
$$

$\frac{k}{2}$ where, $P_{p}$ and $P_{q}$ are permutation matrices with dimensions $\frac{k}{2}$ by $\overline{2}$ and $k+1$ by $k+1$, respectively. To construct the sub matrix $M$ we use a centered stencil (Figure 7), which produces the following system

$$
\left[\begin{array}{cc}
\frac{-1}{2} & \frac{1}{2} \\
1 & 1
\end{array}\right]\left[\begin{array}{l}
x_{1} \\
x_{2}
\end{array}\right]=\left[\begin{array}{l}
1 \\
0
\end{array}\right]
$$

and from the solution $\left[x_{1}, x_{2}\right]=[-11]$, we obtain the rows of $M$. Putting all together we get:

$$
G=\frac{1}{\Delta x}\left[\begin{array}{rrrrrr}
\frac{-8}{3} & 3 & \frac{-1}{3} & & & \\
-1 & 1 & & \\
& \ddots & \ddots & \\
& -1 & 1 & \\
& & \frac{1}{3} & -3 & \frac{8}{3}
\end{array}\right]_{(\mathrm{m}+1)(m+2)}
$$

The $2^{\text {nd }}$-order one-dimensional mimetic gradient. For higher orders, our resulting gradient operators differ from CG. Here we present our $4^{\text {th }}$-order $\mathbf{G}$ :

$$
G=\frac{1}{\Delta x}\left[\begin{array}{lllllll}
\frac{-352}{105} & \frac{35}{8} & \frac{-35}{24} & \frac{21}{40} & \frac{-5}{56} & 0 \ldots \\
\frac{16}{105} & \frac{-31}{24} & \frac{29}{24} & \frac{-3}{40} & \frac{1}{168} & 0 & \ldots \\
0 & \frac{1}{24} & \frac{-9}{8} & \frac{9}{8} & \frac{-1}{24} & 0 & \ldots \\
\vdots & 0 & \ddots & \ddots & \ddots & \ddots &
\end{array}\right]_{(\mathrm{m}+1)(\mathrm{m}+2)}
$$

In eqn. (14) we show only the set of rows necessary to illustrate the overall structure of the matrix.

\section{Divergence}

To construct a $k$ th-order mimetic divergence operator we need at least $2 k+1$ cells $(m \geq 2 k+1)$ so that there is no overlapping between $A$ and $A^{\prime}$.
The methodology to construct a mimetic divergence is the same used to construct the mimetic gradient; except that $A$ and $A^{0}$ will have dimensions $\frac{k}{2}-1$ by $\mathrm{k}+1$.As before, we first exhibit the $2^{\text {nd }}$-order operator,

$$
D=\frac{1}{\Delta x}\left[\begin{array}{cccc}
0 & \cdots & \\
-1 & 1 & \\
\ddots & \ddots & \\
& -1 & 1 \\
& & \ldots & 0
\end{array}\right]_{(m+2),(m+1)}
$$

Notice that the first and last rows of eqn. (15) are zero, this is because on one hand, the divergence does not have a physical meaning at the boundary nodes, and on the other hand, formula in eqn. (6) needs $D$ and $G$ to me compatible matrices under addition, and $G^{t}$ is $(m+2)$ by $(m+1)$, while $D$ without "augmentation", is $m$ by $(m+1)$. Our resulting divergence operators differ from $C G$ when $k \geq 4$. Here is our $4^{\text {th }}$-order D:

$$
D=\frac{1}{\Delta x}\left[\begin{array}{llllllll}
0 & \cdots & & & & & \\
\frac{-11}{12} & \frac{17}{24} & \frac{3}{8} & \frac{-5}{24} & \frac{1}{24} & 0 & \cdots \\
\frac{1}{24} & \frac{-9}{8} & \frac{9}{8} & \frac{-1}{24} & 0 & \cdots \\
0 & \ddots & \ddots & \ddots & \ddots & \ddots
\end{array}\right]_{(m+2),(m+1)}
$$

again, in eqn. (16), we present only the set of rows necessary to illustrate the overall structure of the matrix.

\section{Weight Matrix P}

The diagonal weight matrix $P$ (in eqn. (6)) is obtained by:

$$
\mathbf{G}^{T} p=b_{m+2}
$$

where $p$ is the main diagonal of $P$, and $b_{m+2}$ is the desired column sum $\left[\begin{array}{lllll}-1 & \cdots & 0 & \cdots & 1\end{array}\right]^{T}$.

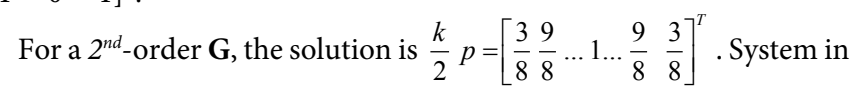
eqn. (17) is over determined but consistent. For our 4 th-order $\mathbf{G}$ (and $m=20)$, we get:

$$
p=\left[\frac{227}{641} \frac{941}{766} \frac{811}{903} \frac{1373}{1348} \frac{1401}{1400} \frac{36343}{36342} \frac{943491}{943490} 1 \ldots\right]^{T}
$$

\section{Weight matrix Q}

The same procedure is applied to get matrix $Q$ (also from in eqn. (6),

$$
\mathbf{D}^{T} q=b_{m+1} \text {, }
$$

System in eqn. (19) is also overdetermined but consistent, and has solution $q=\overrightarrow{1}$ for a $2 n d$-order $\mathbf{D}$. In case of our 4 th-order $\mathbf{D}$ (and $m=20$ ), we get:

$$
q=\left[1 \frac{2186}{1943} \frac{1992}{2651} \frac{1993}{1715} \frac{649}{674} \frac{699}{674} \frac{18170}{18171} \frac{471744}{471745} 1 \ldots\right]^{T}
$$

\section{$2 \mathrm{D}$ and $3 \mathrm{D}$ Operators}

In this section we explain how to construct higher dimensional operators using the ones from and Kronecker products.

To construct a two-dimensional gradient:

$$
G_{x, y}=\left[\begin{array}{l}
S_{x} \\
S_{y}
\end{array}\right]
$$


where,

$$
\begin{aligned}
& S x=\widehat{I}_{n}^{T} \otimes \mathbf{G}_{x}, \\
& S y=G_{y} \otimes \widehat{I}_{m}^{T},
\end{aligned}
$$

$\mathbf{G}_{x^{\prime}} \mathbf{G}_{y}$ are the one-dimensional mimetic gradient operators for $x$ and $y$, respectively. $I_{m}$ denotes an "augmented" identity matrix (first and last rows are zero),

$$
\widehat{I}_{m}=\left[\begin{array}{ccc}
0 & \cdots & \\
1 & & \\
& \ddots & \\
& & 1 \\
& \cdots & 0
\end{array}\right]_{(m+2), m}
$$

To construct a three-dimensional gradient:

$$
G_{x, y}=\left[\begin{array}{l}
S_{x} \\
S_{y} \\
S_{z}
\end{array}\right]
$$

where,

$$
\begin{aligned}
& S_{x}=\widehat{I}_{o}^{T} \otimes \widehat{I}_{n}^{T} \otimes \mathbf{G}_{x}, \\
& S_{y}=\widehat{I}_{o}^{T} \otimes \mathbf{G}_{y} \otimes \widehat{I}_{m}^{T}, \\
& S_{z}=G_{z} \otimes \widehat{I}_{n}^{T} \otimes \widehat{I}_{m}^{T} .
\end{aligned}
$$

Here, $\mathbf{G}_{x^{\prime}} \mathbf{G}_{y^{\prime}} \mathbf{G}_{z}$ are the one-dimensional mimetic gradient operators for $x, y$ and $z$, respectively.

To construct a two-dimensional divergence:

$$
\mathbf{D}_{\mathrm{x}, \mathrm{y}}=\left[\mathrm{S}_{\mathrm{x}}, \mathrm{S}_{\mathrm{y}}\right]
$$

where,

$$
\begin{aligned}
& S_{x}=\widehat{I}_{n} \otimes D_{x}, \\
& S_{y}=D_{y} \otimes \widehat{I}_{n},
\end{aligned}
$$

$\mathbf{D}_{x}, \mathbf{D}_{y}$ are the one-dimensional mimetic divergence operators for $x$ and $y$, respectively.

To construct a three-dimensional divergence:

where,

$\mathrm{D}_{\mathrm{xyz}}=\left[\mathrm{S}_{\mathrm{x}}, \mathrm{S}_{\mathrm{y}}, \mathrm{S}_{\mathrm{z}}\right]$

$S_{x}=\hat{I}_{0} \otimes \hat{I}_{n} \otimes D_{x}$,

$S_{y}=\widehat{I}_{0} \otimes D_{y} \otimes \hat{I}_{m}$,

$S_{z}=D_{z} \otimes \hat{I}_{n} \otimes \hat{I}_{m}$.

$\mathbf{D}_{2} \mathbf{D}_{\gamma}, \mathbf{D}_{z}$ are the one-dimensional mimetic divergence operators for $x, y$ and $z$, respectively.

One-, two- and three-dimensional laplacian operators are obtained by:

$$
\mathbf{L}_{x=} \mathbf{D}_{x} \mathbf{G}_{x}
$$

$\mathbf{L} x y=\mathbf{D} x y \mathbf{G} x y$,

$\mathbf{L} x y z=\mathbf{D} x y z \mathbf{G} x y z$.

Given a three-dimensional vector field:

$$
\mathbf{F}=U \mathbf{i}+V \mathbf{j}+W \mathbf{k},
$$

the curl is defined as,

$$
\Delta \times \mathbf{F}=\left|\begin{array}{ccc}
i & j & k \\
\frac{\partial}{\partial x} & \frac{\partial}{\partial y} & \frac{\partial}{\partial z} \\
U & V & W
\end{array}\right|=\left(\frac{\partial W}{\partial y}-\frac{\partial V}{\partial z}\right) i+\left(\frac{\partial U}{\partial z}-\frac{\partial W}{\partial x}\right) j+\left(\frac{\partial V}{\partial x}-\frac{\partial U}{\partial y}\right) k,
$$

we can express in eqn. (40) as,

$$
\nabla \times \mathbf{F}=\left(\nabla \cdot \mathbf{F}_{y z}^{*}\right) i+\left(\nabla \cdot \mathbf{F}_{z x}^{*}\right) j+\left(\nabla \cdot \mathbf{F}_{x y}^{*}\right) k,
$$

where,

$$
\begin{aligned}
& \mathbf{F}_{y z}^{*}=W j-V k, \\
& \mathbf{F}_{z x}^{*}=U k-W i, \\
& \mathbf{F}_{x y}^{*}=V i-U j .
\end{aligned}
$$

Therefore,

$$
\begin{aligned}
& \mathrm{C}_{x y} \mathbf{F}=\left(\mathbf{D}_{x y} \mathbf{F}_{y z}^{*}\right) i+\left(\mathbf{D}_{z x} \mathbf{F}_{z x}^{*}\right) j+\left(\mathbf{D}_{x y} \mathbf{F}_{x y}^{*}\right) k \\
& \mathrm{C}_{x y z} \mathbf{F}=\left(\mathbf{D}_{y z} \mathbf{F}_{y z}^{*}\right) i+\left(\mathbf{D}_{z x} \mathbf{F}_{z x}^{*}\right) j+\left(\mathbf{D}_{x y} \mathbf{F}_{x y}^{*}\right) k
\end{aligned}
$$

are the two- and three-dimensional mimetic curls, respectively.

\section{Compact Operators}

High-order $(m \geq 4)$ mimetic operators can be represented in a "compact way" by factorizing the original matrices [25]. By doing this, we can attain higher orders of accuracy using only the smallest stencil,

$$
\text { . } \mathbf{D}_{k t h}=\mathbf{D}_{2 n d} \boldsymbol{R}_{k t h}
$$

where $R_{k t h}$ denotes the right factor matrix that when multiplied by the $2 n d$-order divergence produces a $k t h$-order $\mathbf{D}$ operator. The same can be done for the gradient,

$$
\mathbf{G}_{k t h}=L_{k t h} \mathbf{G}_{2 n d}
$$

the reason why we factorize the divergence from the right and the gradient from the left is because in this way we can express the laplacian operator as follows:

$$
\mathbf{L}_{k t h}=\mathbf{D}_{2 n d} R_{k t h} L_{k t h} \mathbf{G}_{2 n d} \text {. }
$$

$\mathbf{L}_{k t h}$ is our $k t h$-order mimetic laplacian, and $R_{k t h} L_{k t h}$ is called the "star" operator (S). $\mathbf{S}$ can be seen as a tensor that contains properties that are inherent to each problem. The authors used compact representation of the CG operators to solve problems of acoustic wave propagation [6].

\section{Accuracy Tests}

We performed several accuracy tests to compare our fourth order operators with those defined [7]. In this section we show two of those tests in Figure 8,

\section{$F(x)=\log x+\cos x$}

The following tables show the magnitude of the error obtained with each method (Castillo-Grone and Corbino-Castillo) (Table 1).

Testing the laplacians with an elliptic problem (Table 2)

$\nabla^{2} f(x)=e^{x}$,

Subject to:

$$
\begin{aligned}
& \alpha f(0)-\beta f^{\prime}(0)=0, \\
& \alpha f(1)+\beta f^{\prime}(1)=2 e
\end{aligned}
$$

with $\alpha=1$ and $\beta=1$. We obtained the following results.

As shown on Table 3 and Figure 9, the new mimetic laplacian 
Citation: Corbino J, Castillo JE (2018) High Order Mimetic Finite Difference Operators Satisfying a Gauss Divergence Theorem. J Appl Computat Math 7: 387. doi: 10.4172/2168-9679.1000387

Page 5 of 6

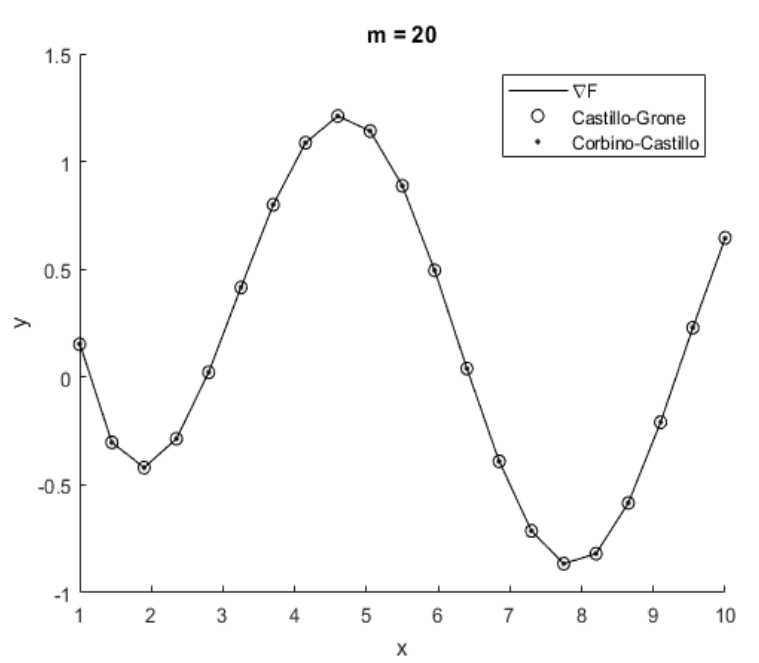

Figure 8: Comparison between continuous and discrete solutions. Visually, both discrete solutions are identical.

\begin{tabular}{|c|c|c|c|}
\hline & \multicolumn{2}{|c|}{ Gradient } & \\
\hline $\boldsymbol{m}$ & $\boldsymbol{C G \| E \| _ { 2 }}$ & $\boldsymbol{C G}\|E\|_{2}$ & $(\Delta \boldsymbol{x})^{\mathbf{4}}$ \\
\hline 10 & 0.0915 & 0.0204 & 0.6561 \\
\hline 20 & 0.0066 & 0.0047 & 0.0410 \\
\hline 40 & 0.0019 & $8.7 \mathrm{e}-4$ & 0.0026 \\
\hline 80 & $2.6 \mathrm{e}-4$ & $1.0 \mathrm{e}-4$ & $1.6 \mathrm{e}-4$ \\
\hline
\end{tabular}

Table 1: Comparing divergences.

\begin{tabular}{|c|c|c|c|}
\hline & \multicolumn{2}{|c|}{ Gradient } & \\
\hline $\boldsymbol{m}$ & $\boldsymbol{C G \| E \| _ { 2 }}$ & $\boldsymbol{C G}\|E\|_{2}$ & $(\Delta \boldsymbol{x})^{\mathbf{4}}$ \\
\hline 10 & 0.0166 & 0.0174 & 0.6561 \\
\hline 20 & 0.0018 & 0.0018 & 0.0410 \\
\hline 40 & $4.4 \mathrm{e}-4$ & $4.5 \mathrm{e}-4$ & 0.0026 \\
\hline 80 & $5.8 \mathrm{e}-5$ & $5.9 \mathrm{e}-5$ & $1.6 \mathrm{e}-4$ \\
\hline
\end{tabular}

Table 2: Testing the laplacians with an elliptic problem.

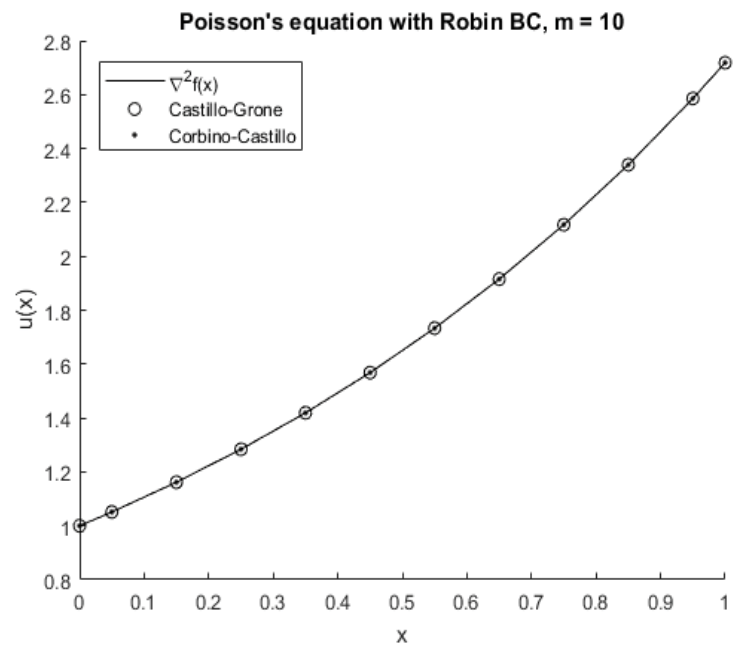

Figure 9: Solution to equation (51). Both approximations look identical.

operator is also considerably more precise than its CG counterpart (in some cases for up to two orders of magnitude).

\begin{tabular}{|c|c|c|c|}
\hline & \multicolumn{2}{|c|}{ Gradient } & \\
\hline $\boldsymbol{m}$ & $\boldsymbol{C G \| E \| _ { 2 }}$ & $\boldsymbol{C G}\|E\|_{2}$ & $(\Delta \boldsymbol{x})^{\mathbf{4}}$ \\
\hline 10 & $4.0 \mathrm{e}-5$ & $1.2 \mathrm{e}-6$ & 0.6561 \\
\hline 20 & $4.3 \mathrm{e}-6$ & $6.8 \mathrm{e}-8$ & 0.0410 \\
\hline 40 & $4.1 \mathrm{e}-7$ & $9.5 \mathrm{e}-9$ & 0.0026 \\
\hline 80 & $3.8 \mathrm{e}-8$ & $1.0 \mathrm{e}-9$ & $1.6 \mathrm{e}-4$ \\
\hline
\end{tabular}

Table 3: The new mimetic laplacian operator.

These tests have been done using the Mimetic Operators Library Enhanced (MOLE) [24].

\section{Conclusions}

High order mimetic finite difference operators that satisfy a discrete extended Gauss-Divergence theorem have been presented. These operators have the same order of accuracy in the interior as well as the boundary, no free parameters and optimal bandwidth. They are constructed on staggered grids, using weighted inner products with a diagonal norm. Their construction using linear algebra illustrate the clarity of their formulation. A compact formulation, which uses the minimum second order stencils, has also been presented. These operators have been implemented in the open source mathematical library MOLE. Mimetic finite difference schemes using this operator produce excellent results on our test cases.

\section{Acknowledgments}

We want to thank Dr. Guillermo Miranda for his useful suggestions on several sections of this article.

\section{References}

1. Kreiss HO, Scherer G (1974) Finite Element and Finite Difference Methods for Hyperbolic Partial Differential Equations. Mathematical Aspects of Finite Elements in Partial Differential Equations, pp: 195-212.

2. Shashkov M (1995) Conservative Finite-Difference Methods on General Grids. CRC Press.

3. Guevara JJ, Arteaga AJ (2007) A Second Order Mimetic Approach for Tracer Flow in Oil Reservoirs. Society of Petroleum Engineers, Proceedings from $X$ Latin American and Caribbean Petroleum Engineering Conference.

4. Guevara JJ, Rojas S, Freites M, Castillo JE (2007) Convergence of a Mimetic Finite Difference Method for Static Diffusion Equations. Advances in Difference Equations.

5. Castillo JE, Hyman JM, Shashkov M, Steinberg S (2001) Fourth- and sixthorder conservative finite difference approximations of the divergence and gradient. Applied Numerical Mathematics 37: 171-187.

6. Cordova LJ, Rojas O, Otero B, Castillo JE (2016) Compact finite difference modeling of 2-D acoustic wave propagation. Journal of Computational and Applied Mathematics 295: 83- 91.

7. Castillo JE, Grone RD (2003) A Matrix Analysis Approach to Higher-Order Approximations for Divergence and Gradients Satisfying a Global Conservation Law. Matrix Analysis and Applications 1: 128-142.

8. Castillo JE, Miranda GF (2013) Mimetic Discretization Methods. CRC Press

9. Rojas O, Day S, Castillo JE, Dalguer LA (2008) Modeling of rupture propagation using high order mimetic finite differences. Geophysical Journal International 172: $631-650$.

10. Abouali M, Castillo JE (2017) Solving Poisson equation with Robin boundary condition on a curvilinear mesh using high order mimetic discretization methods. Mathematics and Computers in Simulation 139: 23-36.

11. Batista E, Castillo JE (2009) Mimetic schemes on non-uniform structured meshes. Electronic Transactions on Numerical Analysis 34: 152-162.

12. Castillo JE, Yasuda M (2005) Linear Systems Arising for Second-Order Mimetic Divergence and Gradient Discretizations. Journal of Mathematical Modeling and Algorithms 4: 67-82.

13. Da Veiga LB, Lipnikov K, Manzini G (2014) The Mimetic Finite Difference Method for Elliptic Problems. Springer. 
Citation: Corbino J, Castillo JE (2018) High Order Mimetic Finite Difference Operators Satisfying a Gauss Divergence Theorem. J Appl Computat Math 7: 387. doi: 10.4172/2168-9679.1000387

14. Hernndez FF, Castillo JE, Larrazbal GA (2007) Large sparse linear systems arising from mimetic discretization. Computers and Mathematics with Applications 53: 1-11.

15. Hyman JM, Morel J, Shashkov M, Steinberg S (2001) Mimetic Finite Difference Methods for Diffusion Equations. Computational Geosciences 6: 333-352.

16. Hyman JM, Shashkov M (2001) Mimetic finite difference methods for Maxwell's equations and the equations of magnetic diffusion. Progress in Electromagnetic Research 32: 89-121.

17. Sanchez E, Paolini C, Blomgren P, Castillo JE (2015) Algorithms for HigherOrder Mimetic Operators. Lecture Notes in Computational Science and Engineering, pp: 425-434.

18. Sanchez JE, Paolini CP, Castillo JE (2014) The mimetic methods toolkit: An object-oriented API for mimetic finite differences. Journal of Computational and Applied Mathematics 270: 308-322.

19. Solano FF, Guevara JJ, Rojas O, Otero B, Rodriguez R (2015) A new mimetic scheme for the acoustic wave equation. Journal of Computational and Applied Mathematics 295: 2-12.
20. De LPJ, Ferrer M, Hanzich M, Castillo JE and Cela JM (2014) Mimetic seismic wave modeling including topography on deformed staggered grids. Geophysics 79: $125-141$.

21. Montilla O, Cadenas C, Castillo JE (2006) Matrix approach to mimetic discretization's for differential operators on non-uniform grids. Mathematics and Computers in Simulation 73: 215-225.

22. Blanco J, Rojas O, Chacon C, Castillo JE (2016) Tensor formulation of 3-d mimetic finite differences and applications to elliptic problems. Electronic Transactions on Numerical Analysis (ETNA) 45: 457-475.

23. Bazan C, Abouali M, Castillo JE, Blomgren P (2011) Mimetic finite difference methods in image processing. Computational and Applied Mathematics 30: 701-720.

24. Corbino J, Castillo JE (2017) MOLE: Mimetic Operators Library Enhanced: The Open-Source Library for Solving Partial Differential Equations using Mimetic Methods.

25. Cordova LJ, Rojas O (2012) High-Order Compact Castillo-Grone's Mimetic Operators. 